\title{
Soma dos maiores autovalores da matriz Laplaciana sem sinal de um grafo
}

\author{
Bruno Dias Amaro Carlile Lavor Jorge Alencar \\ Instituto de Matemática, Estatística e Computação Científica, DMA, IMECC, UNICAMP, \\ 13083-859, Campinas, SP. \\ E-mail: brunoama@gmail.br clavor@ime.unicamp.br jorge.fa.lima@gmail.com
}

\section{Leonardo Lima}

Departamento de Engenharia de Produção, CEFET-RJ

20271-110, Maracanã, RJ.

E-mail: llima@cefet-rj.br

\section{Carla Oliveira}

Escola Nacional de Ciências Estatísticas, ENCE, IBGE

20231-050, Rio de Janeiro, RJ.

E-mail: carla.oliveira@ibge.gov.br

Resumo: Seja $G$ um grafo simples com n vértices e e $(G)$ arestas. Denotamos por $A=A(G)$ a matriz de Adjacência de $G$ e $D=D(G)$ a matriz diagonal da soma das linhas de A, isto é, os graus de cada vértice de $G$. A matriz $Q=Q(G)=D+A$, que vem sendo estudada com grande intensidade nos últimos anos, é chamada de matriz Laplaciana sem sinal do grafo $G$. Sendo $Q$ uma matriz simétrica e semi-definida positiva, seus autovalores são reais e não negativos e podem ser ordenados de forma não crescente como $q_{1} \geq q_{2} \geq \ldots \geq q_{n} \geq 0$. Poucos trabalhos têm estudado a soma dos maiores autovalores da matriz $Q$ e, recentemente, Ashraf et al. [1] conjecturaram que

$$
T_{k}(G)=\sum_{i=1}^{k} q_{i} \leq e(G)+\left(\begin{array}{c}
k+1 \\
2
\end{array}\right) .
$$

É fácil provar que a Conjectura (1) é válida para $k=1, n-1, n$ e Ashraf et al. [1] provaram, recentemente, que a Conjectura (1) é válida para os grafos regulares e para $k=2$ quando $G$ é não regular. Neste trabalho, examinamos alguns resultados disponíveis na literatura e apresentamos alguns avanços na Conjectura (1) para o caso $k=3$.

Palavras-chave: grafos extremais, matriz Laplaciana sem sinal, autovalores.

\section{Introdução}

A teoria dos grafos é um ramo da matemática que estuda as relações entre os objetos de um determinado conjunto. Um grafo é denotado por $G(V, E)$, onde $V$ é um conjunto não vazio de objetos denominados vértices e $E$ é um conjunto de pares não ordenados de $V$, chamado arestas. Estruturas que podem ser representadas por grafos estão em toda parte e muitos problemas de interesse prático podem ser formulados como questões sobre certos grafos [4], [5].

A representação geométrica é extremamente útil na visualização de um grafo. Entretanto, extrair propriedades topólogicas e/ou estruturais de um grafo apenas por visualização torna-se um trabalho não aplicável. Porém, um grafo pode ser convertido em uma forma algébrica via matriz. Quando um grafo é representado na forma matricial, algumas operações e propriedades 
podem ser realizadas. Assim, a partir da Teoria de Grafos, uma área que vem cada vez mais se consolidando é a Teoria Espectral de Grafos.

A Teoria Espectral de Grafos se preocupa com a relação entre as propriedades algébricas do espectro de certas matrizes associadas a grafos, como a matriz de Adjacência, Laplaciana ou Laplaciana sem sinal e a topologia dos mesmos. Os autovalores e autovetores das matrizes associadas a um grafo são os invariantes que formam o autoespaço de grafos. Muitas das demonstrações matemáticas, que relaciona a estrutura de um grafo com seu espectro, podem ser encontradas, por exemplo, nos livros de Norman Biggs [2], Dragoś Cvetković [9] e Chris Godsil [11].

Há, na literatura do tema, uma conjectura proposta por Brouwer e Haemers [3] que associa a soma dos $k$ maiores autovalores da matriz Laplaciana de um grafo $G$ com seu número de arestas mais um fator combinatório que depende do valor $k$ adotado.

Recentemente, Ashraf et al. [1] estenderam a Conjectura proposta por Brouwer e Haemers [3] para a matriz Laplaciana sem sinal de um grafo $G$, isto é,

$$
T_{k}(G)=\sum_{i=1}^{k} q_{i} \leq e(G)+\left(\begin{array}{c}
k+1 \\
2
\end{array}\right) .
$$

Sem muitas dificuldades podemos provar que a Conjectura (2) é válida para $k=1, n-1, n$. Ainda, Ashraf et al. [1] provaram que a mesma é válida para $k=2 \mathrm{e}$, se $G$ for regular, ela é válida para $1 \leq k \leq n$. Neste trabalho examinamos alguns resultados disponíveis na literatura e apresentamos alguns avanços na Conjectura (2) para o caso $k=3$.

\section{A matriz laplaciana sem sinal: soma dos seus autovalores}

Definição 2.1. Seja $G$ um grafo simples com $n$ vértices e $e(G)$ arestas. Denotamos por $A=$ $A(G)$ a matriz de Adjacência de $G$ e $D=D(G)$ a matriz diagonal da soma das linhas de $A$, isto é, os graus de cada vértice de $G$. Definimos as matrizes $L=L(G)=D-A$ e $Q=Q(G)=D+A$ como as matrizes Laplaciana e Laplaciana sem sinal do grafo $G$, respectivamente.

Da Definição acima, temos os seguintes resultados:

Teorema 2.2. [8] Sejam $G$ um grafo com $n$ vértices, $Q$ a matriz Laplaciana sem sinal de $G$ e $q$ um autovetor da matriz $Q$ associado ao autovalor $q_{i}, i=1,2, \ldots, n$. Então:

(i) $Q$ é simétrica e semi-definida positiva, isto é, $q^{t} Q q \geq 0$.

(ii) Os autovalores $q_{i}$ são reais e não negativos.

Sendo $q_{i}$ reais e não negativos, eles podem ser ordenados de forma não crescente como $q_{1} \geq q_{2} \geq \ldots \geq q_{n} \geq 0$.

Teorema 2.3. [8] Se $G$ é um grafo bipartido, então o menor autovalor de $Q$ é zero.

Definição 2.4. Sejam $G_{1}\left(V_{1}, E_{1}\right)$ e $G_{2}\left(V_{2}, E_{2}\right)$ grafos disjuntos com $V_{1}, V_{2}$ e $E_{1}, E_{2}$ seus respectivos conjuntos de vértices e arestas. A união $G_{1} \cup G_{2}$ é o grafo $\left(V_{1} \cup V_{2}, E_{1} \cup E_{2}\right)$. A junção ( "join") $G=G_{1} \vee G_{2}$ é obtida de $G_{1} \cup G_{2}$ e cada vértice de $G_{1}$ é ligado por uma nova aresta a cada vértice de $G_{2}$.

Baseado na Conjectura proposta por Brouwer e Haemers [3] para a matriz Laplaciana L, Ashraf et al. [1] conjecturaram que

$$
T_{k}(G)=\sum_{i=1}^{k} q_{i} \leq e(G)+\left(\begin{array}{c}
k+1 \\
2
\end{array}\right) .
$$


Neste trabalho, estudamos o caso $k=3$, ou seja,

$$
q_{1}+q_{2}+q_{3} \leq e(G)+6 .
$$

Os casos $k=1, k=n-1$ e $k=n$ são imediatos e Ashraf et al. demonstraram em [1] a Conjectura válida para o caso $k=2$.

A partir do uso do AutoGraphiX (AGX), ferramenta baseada na metaheurística Variable Neighborhood Search para geração e descrição de classes de grafos e ainda determinação de grafos extremais (conforme referência [7]), a função $f(G)=m+6-\left(q_{1}+q_{2}+q_{3}\right)$ foi otimizada e a classe de grafos $\mathcal{G}=\overline{\left(K_{n-4} \vee \bar{K}_{2}\right) \cup 2 K_{1}}=K_{2} \vee\left((n-4) K_{1} \cup K_{2}\right), n \geq 4$ foi obtida. A Figura 1 ilustra os grafos da classe $\overline{\left(K_{n-4} \vee \bar{K}_{2}\right) \cup 2 K_{1}}$.

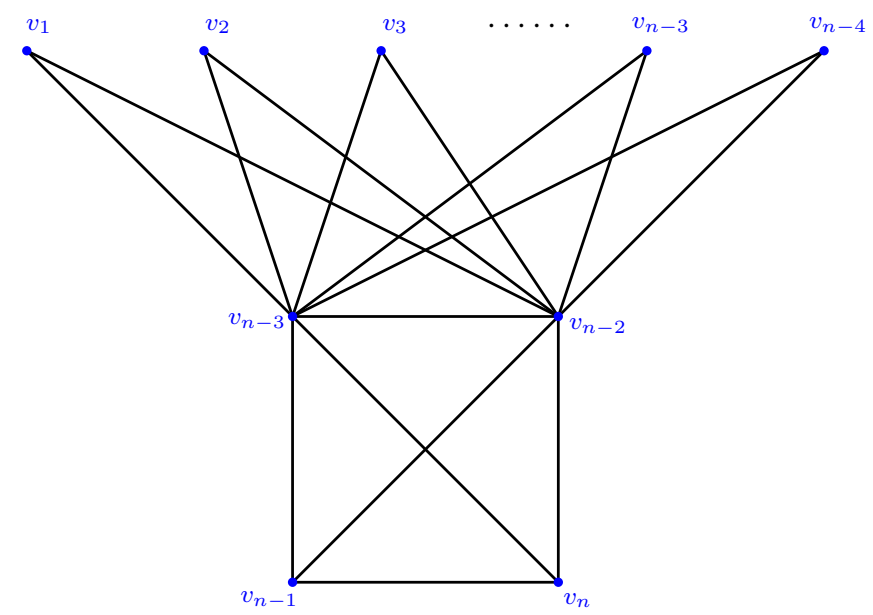

Figura 1: Grafo $\mathcal{G}=\overline{\left(K_{n-4} \vee \bar{K}_{2}\right) \cup 2 K_{1}}=K_{2} \vee\left((n-4) K_{1} \cup K_{2}\right), n \geq 4$

Como o AGX é um procedimento heurístico, não se pode afirmar que a classe de grafos obtida seja ótima, isto é, a função $f(G)$ dada acima fornece o melhor resultado possível. Entretanto, este fato motivou o desenvolvimento dos resultados teóricos a seguir.

Teorema 2.5. Seja o grafo $\mathcal{G}=K_{2} \vee\left((n-4) K_{1} \cup K_{2}\right), n \geq 4$ e $Q(\mathcal{G})$ a matriz Laplaciana sem sinal de $\mathcal{G}$. Então:

(i) 2 é autovalor de multiplicidade $n-4$ em $Q(\mathcal{G})$.

(ii) $n-2$ é autovalor de multiplicidade 1 em $Q(\mathcal{G})$.

Demonstração. Seja o conjunto de vértices $V=\left\{v_{1}, v_{2}, \cdots, v_{n}\right\}$. Mantendo essa ordenação em $V$, temos que a matriz Laplaciana sem sinal $Q(\mathcal{G})$ é dada por

\begin{tabular}{|c|c|c|c|c|c|c|c|c|}
\hline & $v_{1}$ & $v_{2}$ & $\cdots$ & $v_{n-4}$ & $v_{n-3}$ & $v_{n-2}$ & $v_{n-1}$ & $v_{n}$ \\
\hline$v_{1}$ & 2 & 0 & $\ldots$ & 0 & 1 & 1 & 0 & 0 \\
\hline$v_{2}$ & 0 & 2 & $\ldots$ & 0 & 1 & 1 & 0 & 0 \\
\hline$\vdots$ & $\vdots$ & $\vdots$ & $\ddots$ & $\vdots$ & $\vdots$ & $\vdots$ & $\vdots$ & $\vdots$ \\
\hline$v_{n-4}$ & 0 & 0 & $\ldots$ & 2 & 1 & 1 & 0 & 0 \\
\hline$v_{n-3}$ & 1 & 1 & $\cdots$ & 1 & $n-1$ & 1 & 1 & 1 \\
\hline$v_{n-2}$ & 1 & 1 & $\ldots$ & 1 & 1 & $n-1$ & 1 & 1 \\
\hline$v_{n-1}$ & 0 & 0 & $\cdots$ & 0 & 1 & 1 & 3 & 1 \\
\hline$v_{n}$ & 0 & 0 & $\ldots$ & 0 & 1 & 1 & 1 & 3 \\
\hline
\end{tabular}

Assim:

(i) $\mathrm{O}$ conjunto de vetores $\left\{e_{1}, e_{2}, \cdots e_{n-5}, e_{n-4}\right\}$, onde $e_{i}, i=1,2, \ldots n-4$, é o vetor cuja $i$-ésima entrada é igual a 1 e as demais são todas iguais a zero, forma um conjunto de $n-4$ autovetores associados ao autovalor 2 . 
(ii) $\mathrm{O}$ vetor $v=e_{n-3}-e_{n-2}=(\underbrace{0, \ldots, 0}_{n-4 \text { vezes }}, 1,-1,0,0)$ é um autovetor de $Q(\mathcal{G})$ associado ao autovalor $n-2$.

Teorema 2.6. $[3,10]$ Seja $A$ uma matriz quadrada de ordem $n$. Suponhamos que $A$ seja da forma:

$$
A=\left(\begin{array}{cccc}
A_{11} & A_{12} & \cdots & A_{1 k} \\
A_{21} & A_{22} & \cdots & A_{2 k} \\
\vdots & \vdots & \ddots & \vdots \\
A_{k 1} & A_{k 2} & \cdots & A_{k k}
\end{array}\right)
$$

onde $A_{i j}, 1 \leq i, j \leq k$, é uma matriz de ordem $n_{i} \times m_{j}$ tal que suas linhas têm soma constantes iguais a $c_{i j}$. Seja então

$$
R A=\left(\begin{array}{cccc}
c_{11} & c_{12} & \cdots & c_{1 k} \\
c_{21} & c_{22} & \cdots & c_{2 k} \\
\vdots & \vdots & \ddots & \vdots \\
c_{k 1} & c_{k 2} & \cdots & c_{k k}
\end{array}\right)
$$

Então, o polinômio característico de $R A$ divide o polinômio característico de $A$.

Assim, dos Teoremas 2.5 e 2.6, temos o seguinte Corolário:

Corolário 2.7. O polinômio característico associado a matriz reduzida $R Q(\mathcal{G})$ divide o polinômio característico da matriz $Q(\mathcal{G})$, onde

$$
R Q(\mathcal{G})=\left(\begin{array}{ccc}
2 & 2 & 0 \\
n-4 & n & 2 \\
0 & 2 & 4
\end{array}\right)
$$

Do Teorema 2.6 e do Corolário 2.7, temos que os autovalores de $R Q(\mathcal{G})$ são também autovalores de $Q(\mathcal{G})$. Já do Teorema 2.5, temos $n-4+1=n-3$ autovalores conhecidos. O próximo resultado nos garante que os demais 3 autovalores são determinados pela matriz $R Q(\mathcal{G})$ do Corolário 2.7, ou seja, 2 e $n-1$ não são autovalores de $R Q(\mathcal{G})$.

Teorema 2.8. Os 3 autovalores restantes de $Q(\mathcal{G})$ são determinados pela matriz $R Q(\mathcal{G})$.

Demonstração. O polinômio característico de $R Q(\mathcal{G})$ é dado por

$$
p(x)=-x^{3}+(n+6) x^{2}-(4 n+12) x+24 .
$$

Como

$$
\begin{gathered}
p(2)=-8+(n-6) 4-(4 n+12) 2+24 \quad \Leftrightarrow \quad p(2)=-4 n+16 \neq 0, \quad \forall n>4 \mathrm{e} \\
p(n-2)=(n-2)^{3}+(n+6)(n-2)-(4 n+12)(n-2)+24 \Leftrightarrow \\
p(n-2)=n^{3}-9 n^{2}+12 n+28 \neq 0, \quad \forall n \in \mathbb{N},
\end{gathered}
$$

segue que nem 2 nem $n-2$ são autovalores de $R Q(\mathcal{G})$, gerando o resultado.

Teorema 2.9. Para $n \geq 8$, os autovalores $q_{1}, q_{3}$ e $q_{n}$ de $Q(\mathcal{G})$ são as raízes do polinômio característico associado a $R Q(\mathcal{G})$. 
Demonstração. Pelo Teorema 2.5, n-2 é autovalor com multiplicidade algébrica 1 e 2 é autovalor com multiplicidade $n-4$. Os três autovalores restantes, de acordo com o Teorema 2.8, são determinados pela matriz reduzida $R Q(\mathcal{G})$, de polinômio característico $p(x)=-x^{3}+(n+6) x^{2}-$ $(4 n+12) x+24$. Assim, temos:

$$
\begin{gathered}
p(0)=24 \text { e } p(1)=-3 n+17<0, \quad \forall n \geq 6 \\
p(4)=8 \text { e } p\left(4-\frac{3.5}{n}\right)=-20+\frac{525,22-73,5 n}{n^{3}}<0, \quad \forall n \geq 8 \\
p(n+2)=-12 n+16<0 \text { e } p\left(n+2-\frac{4}{n}\right)=16-\frac{32}{n}+\frac{64}{n^{3}}>0
\end{gathered}
$$

Logo, os outros três autovalores de $Q(\mathcal{G})$ (que equivalem aos autovalores de $R Q(\mathcal{G})$ ) pertencem aos intervalos: $[0,1],\left[4-\frac{3.5}{n}, 4\right]$ e $\left[n+2-\frac{4}{n}, n+2\right]$.

Agora, temos que $q_{1} \geq d_{1}+1$ (Cvetković [9]) e que $q_{2} \geq d_{2}-1$ (K.C.Das [6]). Considerando que, no grafo $\mathcal{G}, d_{1}=d_{2}=n-1$, temos:

$$
\begin{array}{r}
q_{1} \geq n \\
q_{2} \geq n-2
\end{array}
$$

Da relação (5), segue que $q_{1} \in\left[n+2-\frac{4}{n}, n+2\right]$. Por outro lado, $q_{2} \leq n-2$, donde temos, junto com a expressão (6), que $q_{2}=n-2$, (que é em $Q(\mathcal{G})$ o autovalor de multiplicidade 1 conforme Teorema 2.5 (ii)), donde $q_{2} \notin[0,1]$ e $q_{2} \notin\left[4-\frac{3.5}{n}, 4\right]$ (o que já era esperado, pois $n-2$ não é autovalor de $R Q(\mathcal{G})$ e, portanto, não pode pertencer a nenhum dos intervalos). Assim, $q_{j} \in\left[4-\frac{3.5}{n}, 4\right]$ para algum $j=3,4,5, \cdots, n$.

Observando que:

- 2 é autovalor de multiplicidade $n-4$ (Teorema 2.5 (i)),

- $2 \notin\left[4-\frac{3.5}{n}, 4\right]$ e $2 \notin[0,1]$,

- $q_{1} \geq q_{2} \geq \cdots \geq q_{n}$,

podemos concluir que $q_{3} \in\left[4-\frac{3.5}{n}, 4\right]$ e $q_{n} \in[0,1]$, seguindo o resultado.

O Teorema 2.9 acima, em resumo, nos diz que:

(i) $q_{1} \in\left[n+2-\frac{4}{n}, n+2\right]$

(ii) $q_{2}=n-2$

(iii) $q_{3} \in\left[4-\frac{3.5}{n}, 4\right]$

(iv) $q_{4}=q_{5}=\cdots=q_{n-1}=2$

(v) $q_{n} \in[0,1]$

e, com essas informações, obtemos o seguinte resultado:

Teorema 2.10. Seja $G$ um grafo isomorfo ao grafo $\mathcal{G}=\left((n-4) K_{1} \cup K_{2}\right) \vee K_{2}$, com $n \geq 8$. Então,

$$
e(G)+6-\frac{7.5}{n}<T_{3}(G)<e(G)+6
$$

Demonstração. Em primeiro lugar, note que $e(G)=2(n-4)+6=2 n-2$. Como

$$
\begin{gathered}
n+2-\frac{4}{n}<q_{1}<n+2 \\
q_{2}=n-2 \\
4-\frac{3.5}{n}<q_{3}<4
\end{gathered}
$$


segue que:

$$
n+2-\frac{4}{n}+n-2+4-\frac{3.5}{n}<q_{1}+q_{2}+q_{3}<n+2+n-2+4
$$

$\mathrm{e}$

$$
2 n+4-\frac{7.5}{n}<T_{3}(G)<2 n+4 .
$$

Porém, $e(G)=2(n-4)+6=2 n-2 \quad \Leftrightarrow \quad e(G)+6=2 n+4$, donde

$$
e(G)+6-\frac{7.5}{n}<T_{3}(G)<e(G)+6 .
$$

Do Teorema 2.10, podemos ver claramente que $T_{3}(G)$ converge para $e(G)+6$, à medida que $n$ tende ao infinito.

Experimentos computacionais mostrararam que entre todos os grafos da família $G_{t}=K_{2} \vee\left((n-(t+2)) K_{1} \cup K_{t}\right), 2 \leq t \leq n-4$, o grafo $\mathcal{G}=K_{2} \vee\left((n-4) K_{1} \cup K_{2}\right)$ é o que melhor se aproxima de $e(G)+6$. Repare que quando $t=2$ temos $G_{2}=K_{2} \vee\left((n-4) K_{1} \cup K_{2}\right)=\mathcal{G}$.

Para mostrar os resultados de forma algébrica, repetindo a linha de raciocínio desenvolvida no grafo $\mathcal{G}$ para o grafo $G_{t}$, podemos definir o polinômio característico de $G_{t}$ em relação matriz $Q\left(G_{t}\right)$ como

$$
p_{G_{t}}(x)=(x-(n-2))(x-2)^{n-t-3}(x-t)^{t-1} \cdot g(x),
$$

onde

$$
g(x)=x^{3}-(n+2 t+2) x^{2}+(2 n t+4 t+4) x-4\left(t^{2}+t\right)
$$

é o polinômio característico da matriz reduzida do grafo $G_{t}$. Após certas manipulações algébricas, obtemos:

$$
\begin{gathered}
q_{1}<n+\frac{t^{2}-5 t+10}{2}-\frac{t-2}{n} \quad n \geq 13 \\
q_{2}=n-2 \\
q_{3}<2 t
\end{gathered}
$$

de tal forma que

$$
q_{1}+q_{2}+q_{3}<2 n-\frac{t^{2}-t+6}{2}-\frac{t-2}{n} .
$$

Porém,

$$
2 n-\frac{t^{2}-t+6}{2}=e\left(G_{t}\right)+6
$$

implicando em

$$
q_{1}+q_{2}+q_{3}<e\left(G_{t}\right)+6-\frac{t-2}{n} \leq e\left(G_{t}\right)+6 .
$$

Baseado nas informações acima, temos o seguinte resultado:

Teorema 2.11. Seja $G$ um grafo isomorfo ao grafo $G_{t}=K_{2} \vee\left((n-(t+2)) K_{1} \cup K_{t}\right), 2 \leq t \leq n-4$ e $n \geq 13$. Então:

(i) $q_{1}+q_{2}+q_{3}<e\left(G_{t}\right)+6$;

(ii) $G_{2}=K_{2} \vee\left((n-4) K_{1} \cup K_{2}\right)$ é o grafo que melhor se aproxima de $e\left(G_{t}\right)+6$. 


\section{Conclusões}

Neste trabalho, uma família de grafos infinita que satisfaz a Conjectura (3), para o caso $k=3$, foi apresentada e provado que dentre todas as famílias de grafos formadas por join de um grafo completo $K_{s}$ e um grafo com $(n-s)$ vértices isolados, a família de grafos $G=K_{2} \vee\left((n-4) K_{1} \cup K_{2}\right)$ é a que tem a soma $q_{1}+q_{2}+q_{3}$ que melhor se aproxima de $e(G)+6$.

\section{Agradecimentos}

Gostariamos de agradecer ao Conselho Nacional de Desenvolvimento Científico e Tecnológico - CNPq e a Fundação de Amparo à Pesquisa do Estado de São Paulo - FAPESP pelo apoio financeiro desse projeto.

\section{Referências}

[1] F. Ashraf, G.R. Omidi and B. Tayfeh-Rezaie, On the sum of singless Laplacian eingevalues of a graph, Linear Algebra and its Applications, 438, (2013), 4539-4546.

[2] N. Biggs, "Algebraic Graph Theory: Second Edition", Cambridge Mathematical Library, 1994.

[3] A.E. Brouwer and W.H. Haemers, Spectra os graphs: Obtido em: http://homepages.cwi.nl/ aeb/math/ipm.pdf.

[4] P.O. Boaventura Netto, "Grafos - Teoria, Modelos, Algoritmos, 4a. edição revista e ampliada", Editora Edgard Bluncher, 2006.

[5] B. Bollobás, "Graph Theory: An Introductory Course", Graduate Texts in Mathematics, Springer-Verlag, 1979.

[6] K.C. Das, On conjectures involving second largest signless Laplacian eigenvalue of graphs, Linear Algebra and its Applications, 432, (2010), 3018-3029.

[7] G. Caporossi, P. Hansen, Variable neighborhood search for extremal graphs: 1 The AutoGraphiX system, Discrete Mathematics, 212, (2000), 29-44.

[8] D. Cvetković, "Spectral theory of Graphs Based on the Signless Laplacian - A quick Outline", Research Report, Mathematical Institute SANU, 2010.

[9] D. Cvetković, M. Doob, H. Sachs, "Spectra of Graphs - Theory and Application"Academic Press Inc., New York, 1980.

[10] M.A.A. Freitas, "Grafos Integrais, Laplacianos Integrais e $Q$-integrais", Tese de Doutorado, Engenharia de Produção, COPPE, UFRJ, 2009.

[11] C. Godsil, G. Royle, "Algebraic Graph Theory", Graduate Texts in Mathematics, 207, Springer, 2000. 